\title{
Secondary metabolite production of Epicoccum sp. isolated from lignite
}

\author{
Michal Kaliňák, Viera Barátová, Emília Gallová, \\ Zuzana Ondrušová, Daniela Hudecová \\ Department of Biochemistry and Microbiology, Faculty of Chemical and Food Technology, \\ Radlinského 9, SK-812 37 Bratislava \\ michal.kalinak@stuba.sk
}

\begin{abstract}
We have extracted secondary metabolites from fossil fungus Epicoccum sp. isolated from lignite and characterized changes in production on various growth media. Chromatographic separation yielded more than 7 fractions that were characterized spectroscopically. One secondary metabolite showed antimicrobial activity. Peaks in the NMR spectra of isolated fractions differ from compounds described in literature that were isolated from recent strains of Epicoccum. The remaining water phase after the extraction was shown to have protein staining properties and could be also used for fluorescent viability stain of yeast cells.
\end{abstract}

Keywords: Epicoccum, secondary metabolites, pigments, isolation

\section{Introduction}

Secondary metabolites from various organisms serve as a source of potentially useful compounds for pharmacy, diagnostics and other fields. The search for new compounds fuelled by the development of drug resistance and the need for innovation leads researchers to look in extreme locations or unusual places (Pikuta et al. 2007). There have been microorganisms isolated from sites such as glaciers, mountain tops, sea valleys, hot springs or salt lakes (Gostincar et al. 2010).

It is well known that fungi produce a wide range of secondary metabolites. Despite a limited number of enzymes involved in the production, the number of possible metabolites is enormous and it differs even in closely related species (Studt et al. 2012). Moreover, the environmental and nutritional factors influence the range and amount of produced secondary metabolites.

Species Epicoccum is known to produce heterocyclic compounds with (thio)diketopiperazine backbone like epicoccins, difenylalazines or epicorazines, the latter having antibacterial properties (Deffieux et al. 1978a, 1978b; Guo et al. 2009; Wang et al. 2010). Moreover, a fluorescent product named epicocconone was introduced into biochemical laboratories as a sensitive and specific reagent for protein determination and visualization (Bell and Karuso 2003).

Pokorný with colleagues have isolated bacterial and fungal species from lignite excavated from Záhorie coal mine (Pokorný et al. 2005). We used the isolate $9 \mathrm{~b}$ that was shown to be related to recent Epicoccum nigrum for screening for the secondary metabolite production. We have narrowed down the fraction that contains antibacterial compound.
This work characterizes isolated fractions and also deals with the changes in the spectrum of secondary metabolites that are influenced by the medium composition and time evolution. Finally, we have found that the medium left over after the extraction has possible applications in staining proteins and yeast cells.

\section{Experimental}

Epicoccum nigrum i9b (Pokorný et al. 2005) was obtained from the local collection of microorganisms. It was cultured in liquid Czapek-Dox (Cz-D) medium supplemented with yeast extract $\left(5\right.$ g. $\left.\mathrm{L}^{-1}\right)$. For secondary metabolites production $400 \mathrm{ml}$ of media were inoculated with 4 discs cut out from the colony periphery of inoculated $\mathrm{CzD}$ agar plates and let grow for 4 weeks on a rotary shaker at $25^{\circ} \mathrm{C}$ in the dark.

After filtering out mycelium, secondary metabolites from medium were extracted with $100 \mathrm{ml}$ of ethyl acetate (Microchem, Pezinok, Slovakia). After $2 \mathrm{~h}$ shaking, phases were separated and the aqueous phase was extracted two more times with the same amount of ethyl acetate. The organic phases were pooled, anhydrous sodium sulfate was added and after its removal by filtration the filtrate was reduced by vacuum evaporation. The remaining water phase was filtered through a filter paper (Filtrak 388, Spezialpapierfabrik Niederschlag, Germany, wide pores) and GF/A glass fiber filter (Whatman) and used in some assays.

Alternatively, solidified Czapek-Dox agar (CzDA) medium and other media were used. Rye extract agar (RYE) was made by boiling $100 \mathrm{~g}$ of rye grains in water for $30 \mathrm{~min}$. After removing the solids by filtration, $5 \mathrm{~g}$ of glucose and $15 \mathrm{~g}$ of agar were 
added, filled up to $1 \mathrm{~L}$ and sterilized. Oat extract agar (OAT) was made similarly but only $30 \mathrm{~g}$ of oat flakes and no glucose was used. CzDA, potato dextrose agar (PDA) and Sabourad glucose agar (SGA) media were prepared from ready-made powders by Biolife Italy S.l.r., Milan, Italy. Fungus was cultivated on top of agar plates covered with cellophane disks. After cultivation cellophane with mycelium was removed and medium was extracted with chloroform-methanol (2:1). Solid parts were removed by filtration and the filtrate was used for analysis.

Extracts were separated on a thin-layer chromatography plates (Silufol or Silufol UV254, Kavalier, Czechoslovakia) or on preparative $2 \mathrm{~mm}$ plates PSC Kieselgel 60 F254 (Merck) with chloroform:methanol $(94: 6 \mathrm{v} / \mathrm{v})$ and detected under UV light. Several spots of the same color were scraped from the plate, extracted into methanol and concentrated by solvent evaporation. Remains were dissolved in $50 \mu \mathrm{l}$ of deuterated methanol and used for NMR measurements.

The fluorimetric measurements were done on Fluoromax4 (Horiba Yvon-Jobin) controlled by FluorEssence $^{\mathrm{TM}}$ software. Emission spectra from 400 to $550 \mathrm{~nm}$ were measured on fractions from TLC diluted to $2 \mathrm{ml}$ with ethyl acetate with excitation wavelength set to $350 \mathrm{~nm}$. All spectra were corrected by subtracting solvent spectrum and normalized to show relative intensities.

The bioautography was done by putting the TLG strip on top of Mueller-Hinton agar (BioLife Italy) inoculated with $10^{6}$ cells/ml of Bacillus subtilis CCM-1718 for $20 \mathrm{~min}$. The inhibition zones were detected after $5 \mathrm{~h}$ by spraying $0.5 \%$ 2,3,5-triphenyltetrazolium chlorid on top of agar.

For HPLC analysis $5 \mu$ injection was separated on two in-line silica gel columns (Separon SGX $150 \times 3 \mathrm{~mm} 7 \mu \mathrm{m}$, Tessek, Czech republic) with $0.1 \mathrm{ml} . \mathrm{min}^{-1}$ flow of butanol:acetic acid:water (4:1:1) using a Varian system with photodiode array detector and $250 \mathrm{~nm}$ and $370 \mathrm{~nm}$ as reference wavelengths.

NMR spectroscopy was done on $600 \mathrm{MHz}$ Varian NMR system equipped with gHX Nano probe spinning at 1200 or $1500 \mathrm{~Hz}$. Standard proton NMR spectra were acquired with s2pul sequence -45 degree pulse, 2.7 s repetition and 256 transients or $1 \mathrm{D}$ tnnoesy with $1 \mathrm{~s}$ presaturation, calibrated 90 degree pulses, 5 s repetition and $100 \mathrm{~ms}$ mixing time.

SDS PAGE was done in $10 \%$ gel with ovalbumin (Sigma) as standard (Laemmli 1970). Staining with Epicoccum extracts was done for $30 \mathrm{~min}$ without destaining. Staining with Coomassie Brilliant Blue R250 (Sigma) was done for 5 min with subsequent destaining.
Cells of S. cerevisiae for fluorescent microscopy were cultured in YPD medium, centrifuged, washed and resuspended in physiological saline. To $200 \mu \mathrm{l}$ of cell suspension $50 \mu \mathrm{l}$ of water phase after extraction or 2 drops of $0.01 \%$ methylene blue solution were added and suspension was mounted on a microscopy slide. When needed cells were heat killed by passing the slide over a flame 3 times. Microscopy was performed on a Carl Zeiss AxioImager A1 equipped with AxioCam MRc and XCite light source. Filters Fs05 (excitation 395-440 nm, emission $470 \mathrm{~nm}$ ) and PJ (excitation 530-585 $\mathrm{nm}$ emission $615 \mathrm{~nm}$ ) were used for imaging of cells stained with extract and methylene blue, respectively.

\section{Results}

The fungus Epicoccum nigrum i9b was cultured on liquid Czapek-Dox medium supplemented with yeast extract in an attempt to isolate and identify new secondary metabolites from new sources. The fungus produces dark red (almost black in appearance) pigments into the liquid medium. To look more closely on the production of secondary metabolites ethyl acetate (EthAc) was used for extraction. Dark pigment remained in the water phase where also gel-like aggregates, presumably of polysaccharides, precipitated and were difficult to filter out. EthAc extracts had yellow color.

Concentrated extracts were separated on thin-layer chromatographic silica gel plates with various mobile phases. Mixtures of chloroform and methanol (96:4) and toluene, ethyl acetate and formic acid (5:4:1) worked best for separation of metabolites, each being able to resolve different spots (Fig. 2). Finally chloroform - methanol was used to separate metabolites on analytical and later on preparative TLC because these solvents could be removed afterwards and least affect NMR analysis. Although many more bands could be seen only the 7 most abundant and reasonably separated were extracted from TLC (Fig. 1). Under UV light they have blue, yellow, orange or green color.

The first analysis meant to characterize fluorescent properties of isolated secondary metabolites. It is interesting that all of the metabolite have fluorophores. The emission spectra show clear differences between fractions (Fig. 3 left) with band 6 having strong overlap with neighboring bands. Other bands have relatively sharp maxima and only few possible impurities. The band that did not move from the start is also very likely a mixture of compounds and has highest fluorescent intensity (Fig. 3 right).

Next we proceeded with NMR analysis. The amounts obtained from preparative TLC were still tiny so we used probe that can detect $40 \mu \mathrm{l}$ volumes. 


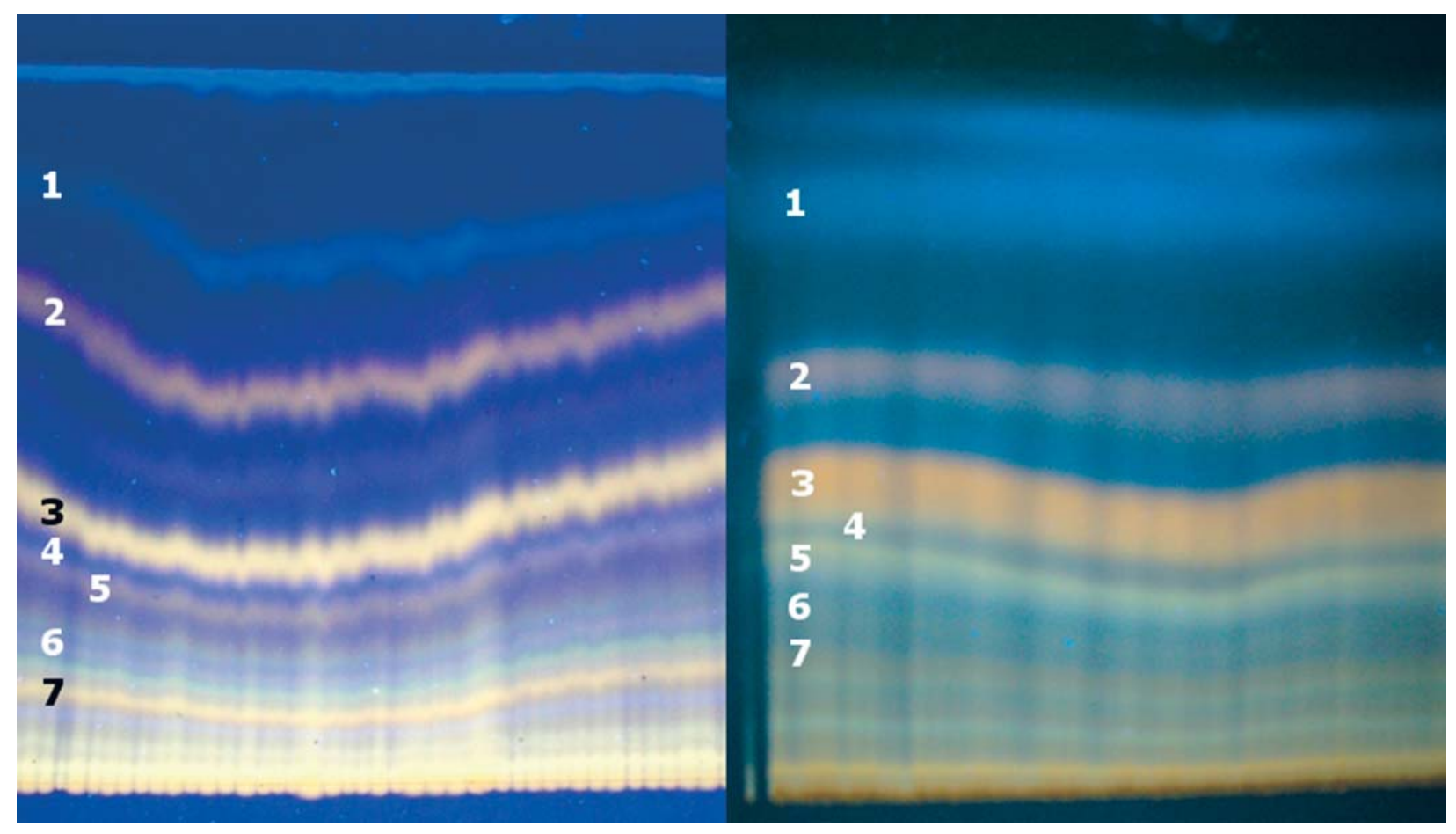

Fig. 1. Analytical (left) and preparative (right) TLC with separated EthAc extracts from Epicoccum nigrum i9b.

Numbers show fractions that were scraped out and used for further analysis.

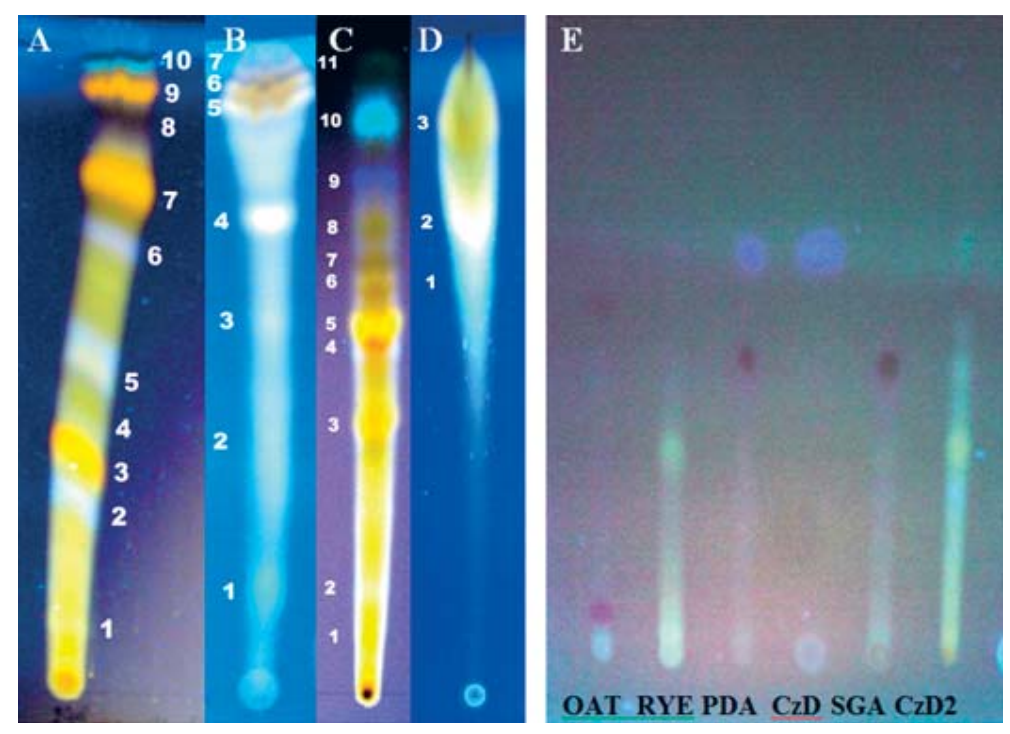

Fig. 2. A-D Separation of EthAc extract from submerged cultivation of E. nigrum i9b in Cz-D medium with mobile phase (A) chloroform:methanol (96:4) (B) ethylacetate:methanol:water (10:1, 35:1)

(C) toluene:ethylacetate:formate (5:4:1) and (D) butanol:acetic acid:water (4:1:1). Numbering was used for work with individual spots and is not used further in this article. (E) Separation and comparison of extracts from various solidified media. See Experimental for media abbreviations. $\mathrm{CzD} 2$ is extract obtained before the fungus lost its pigment producing capacity.

Since the whole volume is in the receiver coil and there is a change in magnetic susceptibility at the edges it has to be spun fast at magic angle so as to remove linebroadening. This causes spinning sidebands to appear in the spectrum at distances equal to spinning speed. The tallest peaks were assigned to solvents and only the small peaks that were approximately hundred times smaller belonged to the compound (Fig. 4). Spinning sidebands and ${ }^{13} \mathrm{C}$ satellites of solvent peaks were thus taller than compound peaks. This precluded measuring good multidimensional spectra even with solvent sup- 


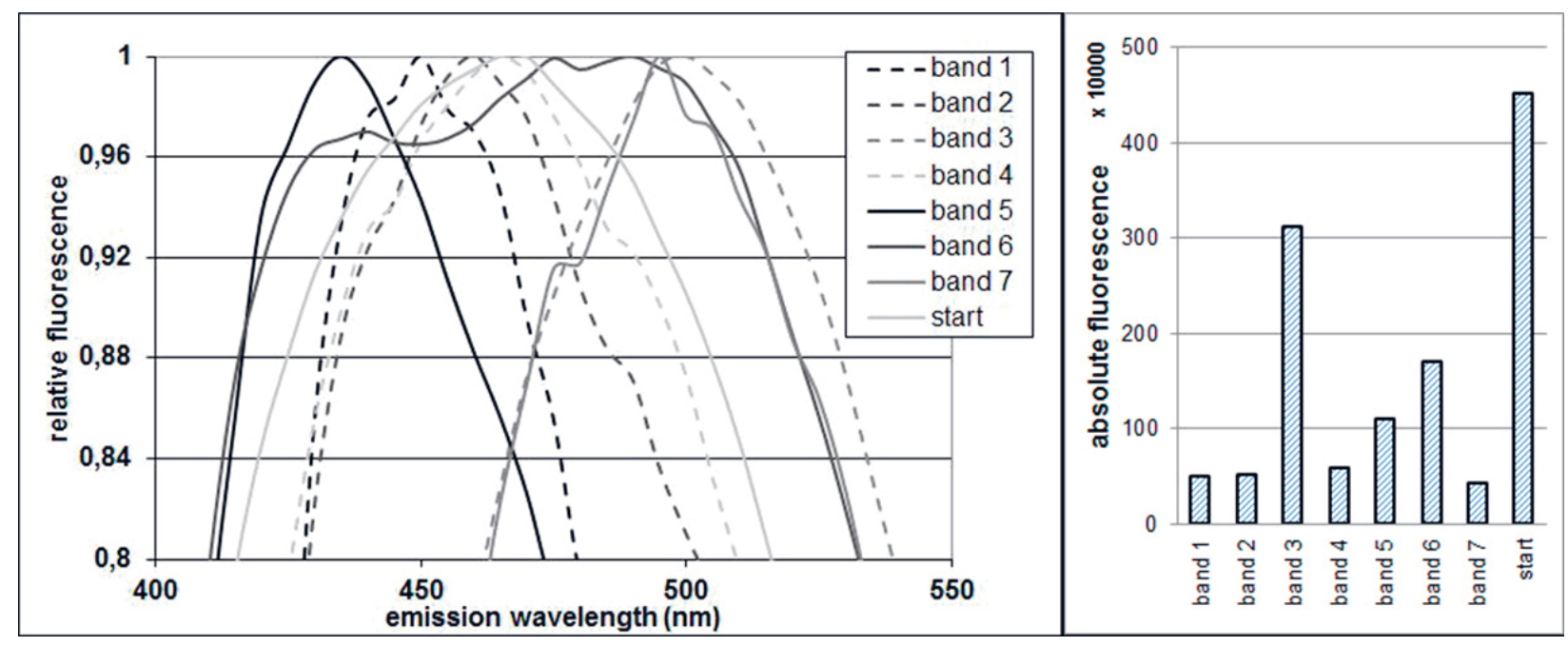

Fig. 3. Normalized emission spectra (left) and fluorescent intensities at maximum peak (right) measured with excitation of $350 \mathrm{~nm}$.

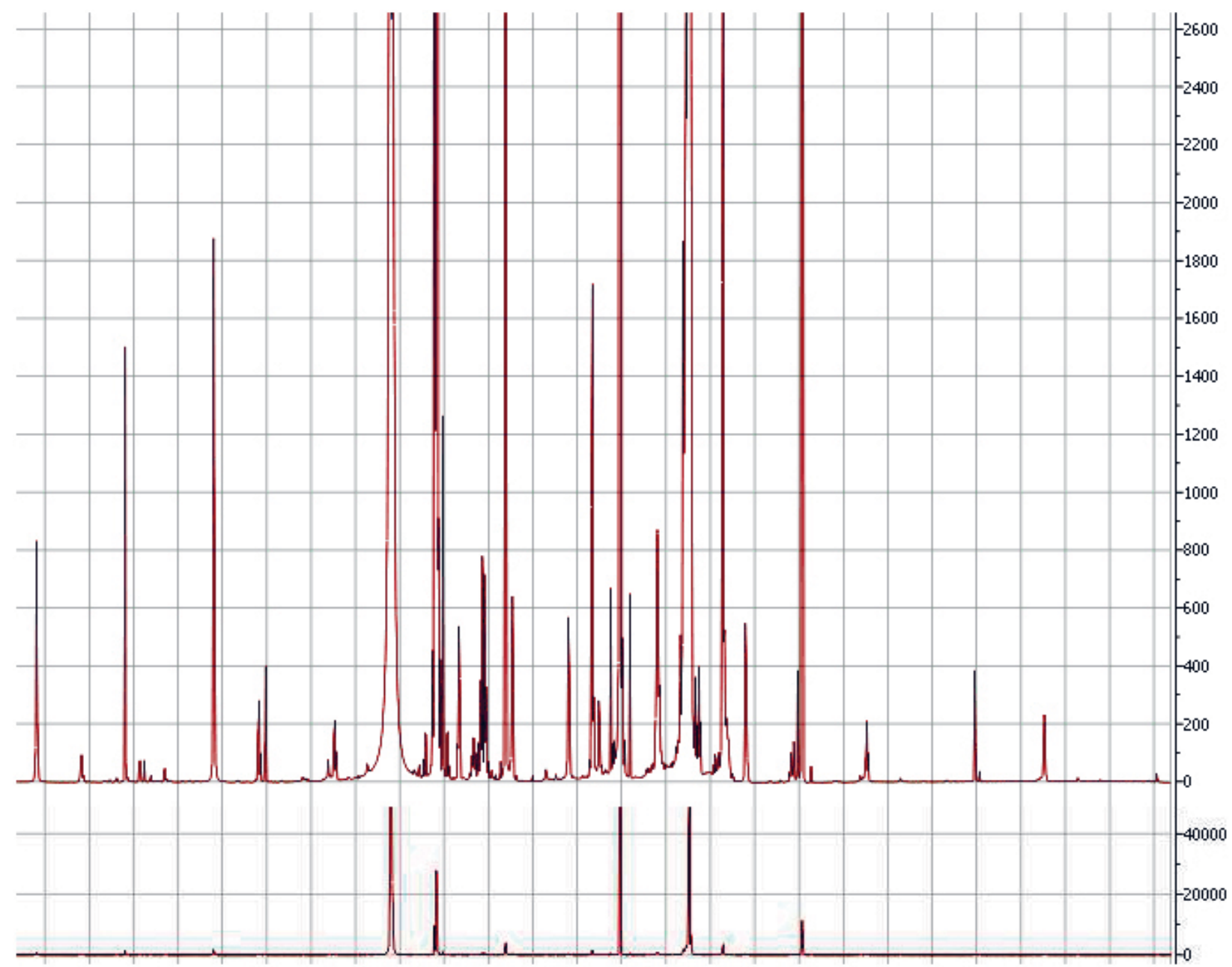

Fig. 4. ${ }^{1} \mathrm{H}$ NMR spectrum of band 3 in deuterated methanol. Lower trace is from the full scale spectrum where the solvent peaks are cropped in approx. third of the full height, upper trace is $\sim 50 \times$ expanded where most tall peaks are spinning sidebands.

pression and therefore hindered the identification. We were, however, able to resolve chemical shifts of several compound peaks and compare them to the ones already published or were predicted from published structures by MNova NMR Predict module (Mestrelab Research S. L., Spain). Chemi- 
cal shifts of epicorazine $\mathrm{A}$ and $\mathrm{B}$, epicocconone, 13 different thiodiketopiperazines as well as 4 diketopiperazines were used but no match was found and in regions where there should have been peaks there were none. Appearance of spectra suggested and subsequent repeated TLC analysis confirmed that some of the extracted bands contained more than one metabolite.

Previous work showed that the fungus produces secondary metabolites having antibacterial properties (Pokorný et al. 2005). We have tried to identify which of the produced metabolite has these properties. Bioautography showed a spot with Rf 0.34 to have antibacterial action on Bacillus subtilis (Fig. 5). After several passages on agar $\mathrm{Cz}-\mathrm{D}$ media the fungus started to lose its pigment production capabilities and only a yellow pigment was released into the medium. The cultivation on PDA and RYE agar media restored the pigment production to certain extent although after 7 days the pigments did not

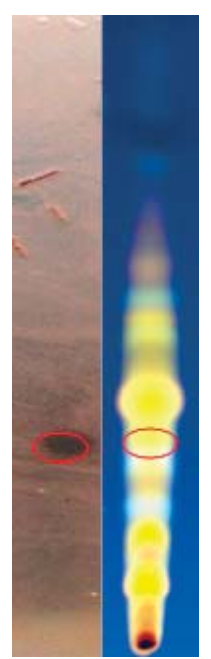

diffuse into the medium (Fig. 6, bottom row). The mycelial patterns also differed considerably, sometimes concealing the pigment production, which could be only seen from the bottom of Petri dish (Fig. 6, top row).

TLC and HPLC analysis showed that Epicoccum produced different metabolites on different media types (Fig 2E, 7).

Based on published results about epicocconone and because of fluorescent properties of our extracted metabolites we also attempted to find whether they would have possible applications in protein determination and visualization or cell staining. Although the pigments extracted with EthAc did not stain gels, the resulting water phase stained proteins with faint yellow color (Fig. 8 left). Staining with Epicoccum metabolites had a lesser contrast than standard Coomassie Brilliant Blue R250 stain (Fig. 8 right). The fluorescence microscopy of yeast cells (S. cerevisiae) stained with the same water phase showed that metabolically inactive cells (heat-killed cells and ascospores) showed green fluorescence which was confirmed with methylene blue staining (Fig. 9).

\section{Discussion}

Epicoccum sp. strain 9b isolated from lignite is according to the ITS sequence closely related but not equal to recent Epicoccum nigrum strains (Pokorný et al. 2005). We have isolated and separated several secondary metabolites from fossil fungus Epicoccum nigrum $\mathrm{i} 9 \mathrm{~b}$ that also show different fluorescent properties. One of the metabolites with the $\mathrm{Rf}$ of 0.34 also had antibacterial properties. The ${ }^{1} \mathrm{H}$ NMR spectra were swamped by the residual solvent peaks

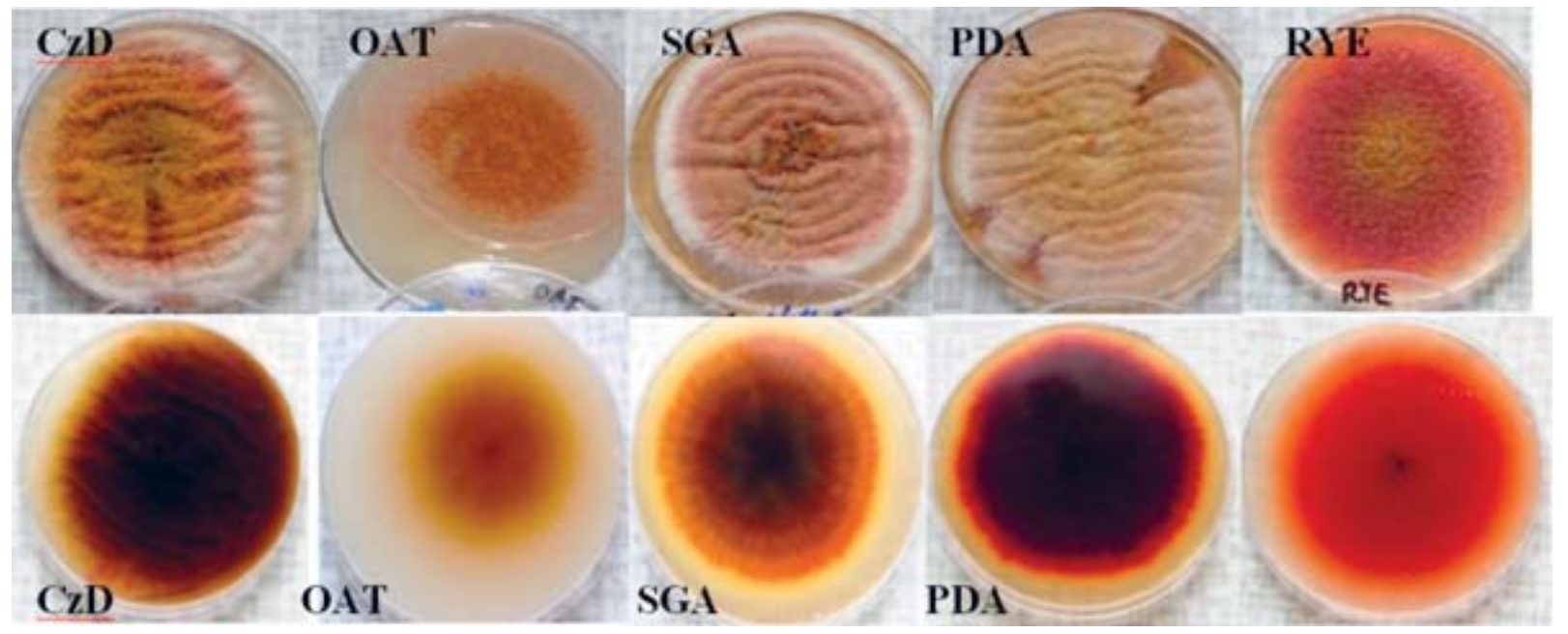

Fig. 6. Comparison of E. nigrum i9b growth and pigment production after 7 days on various types of media. Top row: view from the top showing mycelial patterns.

Bottom row: view from the bottom showing pigmentation of the mycelium. 


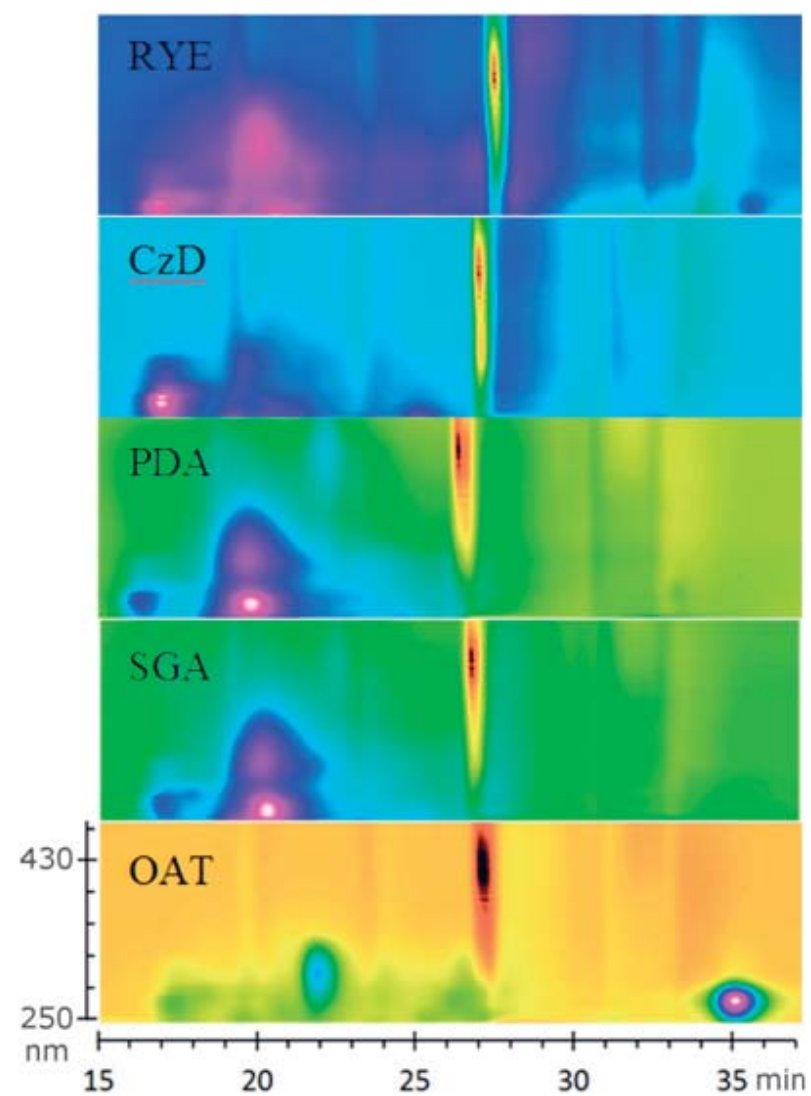

Fig. 7. HPLG PDA chromatograms of E. nigrum i9b EthAc extracts. Shown is only the part of chromatogram containing peaks (15-37 min and $250-475 \mathrm{~nm})$. Note that black peak around $28 \mathrm{~min}$ is negative.
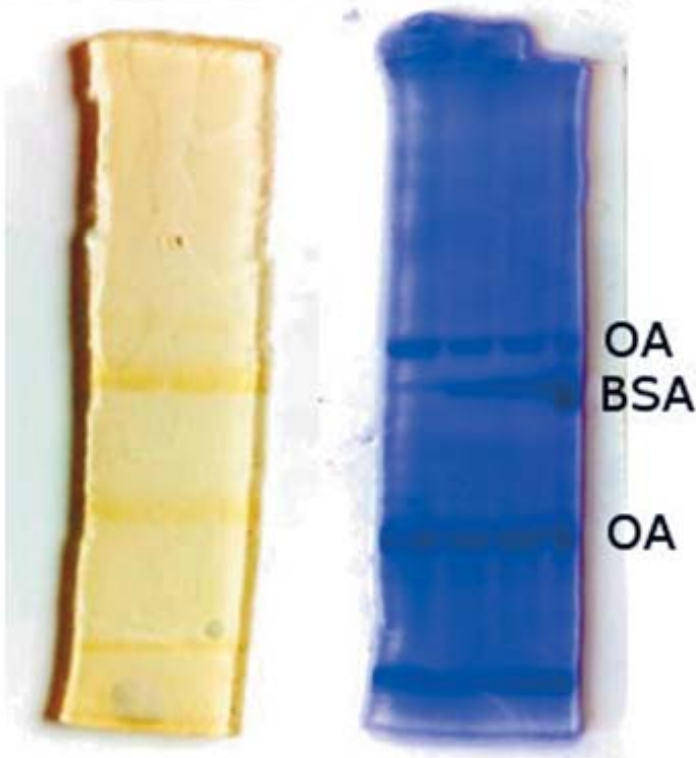

Fig. 8. Bands of ovalbumin (OA and its aggregates) stained with $E$. nigrum water phase after extraction (left) and with Coomassie Brilliant Blue R250 (right). The gel on the right contains also bands of bovine serum albumin (BSA) for verification purposes that are not present in the gel on the left.
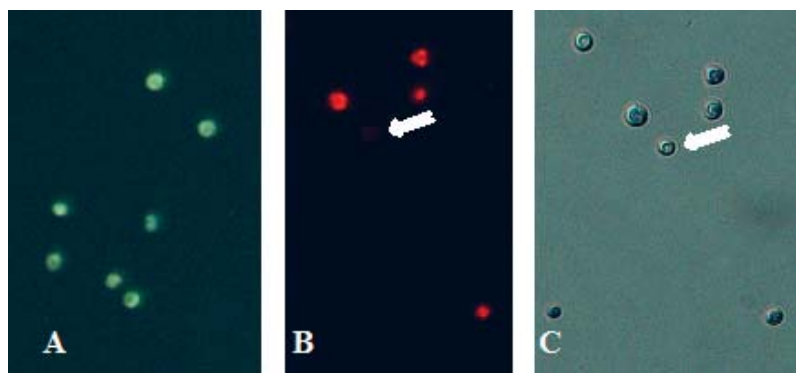

Fig. 9. Vegetative cells and ascospores of $S$. cerevisiae at various fluorescent filters (A filter Fs05, B filter $\mathrm{PJ}$ ) and using the DIC contrast (C). The cells were stained with $E$. nigrum water phase after extraction (A) and with methylene blue (B, C). Arrow shows an unstained viable yeast cell.

with their sidebands also concealing the compound peaks. Therefore we do not have their definitive identification yet, but based on comparison with known and predicted chemical shifts they are different from the ones described previously for this genus. Larger scale isolation will be needed to overcome this drawback.

The microscopic images did not show any changes (not shown) but growth on various solid media revealed differences in colony color and pigmentation (Fig. 6). The fungus lost its pigment formation capabilities after several passages on rich Czapek-Dox medium supplemented with yeast extract although it still produced some of the other metabolites. The production of secondary metabolites before the loss was comparable to the production on RYE medium afterwards (Fig. 2E) which hints on the environmental pressure with the nutrient/stress status being possible causes of such behavior. Similar behavior was observed in Cryptococcus neoformans that lost melanin production capabilities as well as virulence when passaged on rich media, which correlated with disomy at chromosome 13 ( $\mathrm{Hu}$ et al. 2011). The original phenotype is often obtained by cultivation at suboptimal conditions as was the case with RYE medium. Thus, genome plasticity is a phenomenon that needs greater attention from the scientific community also in regards to secondary metabolite production.

The range of produced metabolites depends considerably on the medium composition (Fig. 2E). Glucose containing media (SGA and PDA) showed comparable metabolite production. OAT medium caused the production of such compounds that were not present in other media.

Fluorescent metabolites of Epicoccum nigrum are known to bind to proteins and are used for sensitive protein determination and visualization of protein bands on polyacrylamide gels (Coghlan et 
al. 2005). We have performed similar experiments with EthAc extracts but protein binding was insufficient or slow for protein determination studies (not shown). However, the polyacrylamide gel with separated standard proteins could be visualized with pigments that remained in the water phase after the extraction (Fig. 8). The disadvantage is that the color of the bands is yellow which does not give sufficient contrast compared to Coomassie Brilliant Blue staining. The staining takes longer to saturate the bands but no destaining step is necessary. Visualization under UV light was not successful. It is however possible that chemical modification of the dye could improve the spectral properties of the dye.

There are several dyes used for testing cell viability e.g. methylene blue, trypan blue or methylene violet. We have shown that water phase after the EthAc extraction is suitable for fluorescent staining of dead cells or dormant ascospores (Fig. 9). The mechanism of staining was not investigated but the images show good correlation with methylene blue staining.

\section{Conclusion}

Epicoccum nigrum i9b isolated from lignite produces different secondary metabolites than recent strains. The structure of these compounds remains to be elucidated, but the practical aspects of their properties can be exploited.

\section{Acknowledgement}

This work was supported by the Slovak Research and Development Agency (APVV-0282-10) and the Agency of the Ministry of Education, Science, Research and Sport of the Slovak Republic for the Structural Funds of EU (project ITMS 26240120028).

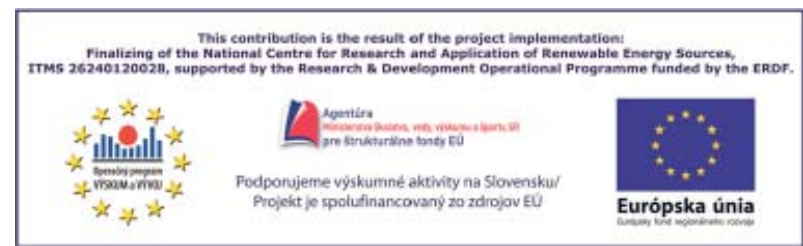

\section{References}

Bell PJL, Karuso P (2003) Journal of the American Chemical Society 125(31): 9304-9305.

Coghlan DR, Mackintosh J, Karuso P (2005) Organic letters 7(12): 2401-2404.

Deffieux G, Baute MA, Baute R, Filleau MJ (1978a) The Journal of antibiotics 31(11): 1102-1105.

Deffieux G, Filleau MJ, Baute R (1978b) The Journal of antibiotics 31(11): 1106-1109.

Gostincar C, Grube M, De Hoog S, Zalar P, GundeCimerman N (2010) FEMS microbiology ecology 71(1): 2-11.

Guo H, Sun B, Gao H, Chen X, Liu S, Yao X, Liu X, Che Y (2009) Journal of natural products 72(12): 2115-2119.

Hu G, Wang J, Choi J, Jung WH, Liu I, Litvintseva AP, Bicanic T, Aurora R, Mitchell TG, Perfect JR, Kronstad JW (2011) BMC genomics 12: 526.

Laemmli UK (1970) Nature 227(5259): 680-685.

Pikuta EV, Hoover RB, Tang J (2007) Critical reviews in microbiology 33(3): 183-209.

Pokorný R, Olejníková P, Balog M, Zifčák P, Hölker U, Janssen M, Bend J, Höfer M, Holiencin R, Hudecová D, Varečka L' (2005) Research in microbiology 156(9): 932-943.

Studt L, Troncoso C, Gong F, Hedden P, Toomajian C, Leslie JF, Humpf H-U, Rojas MC, Tudzynski B (2012) Fungal genetics and biology: $F G$ \& B 49(7): 567-577.

Wang J-M, Ding G-Z, Fang L, Dai J-G, Yu S-S, Wang Y-H, Chen X-G, Ma S-G, Qu J, Xu S, Du D (2010) Journal of natural products 73(7): 1240-1249. 